\title{
Non-parametric Mann-Kendall Test Statistics for Rainfall Trend Analysis in Some Selected States within the Coastal Region of Nigeria
}

\author{
Ihimekpen Ngozi Isioma, Ilaboya Idowu Rudolph, Awah Lauretta Omena \\ Department of Civil Engineering, University of Benin, Benin City, Nigeria \\ Email address: \\ n.ihimekpen@uniben.edu (I. N. Isioma), rudolph.ilaboya@uniben.edu (I. I. Rudolph), ome1602@yahoo.com (A. L. Omena) \\ To cite this article: \\ Ihimekpen Ngozi Isioma, Ilaboya Idowu Rudolph, Awah Lauretta Omena. Non-parametric Mann-Kendall Test Statistics for Rainfall Trend \\ Analysis in Some Selected States within the Coastal Region of Nigeria; Journal of Civil, Construction and Environmental Engineering. \\ Vol. 3, No. 1, 2018, pp. 17-28. doi: 10.11648/j.jccee.20180301.14
}

Received: January 16, 2018; Accepted: February 5, 2018; Published: March 15, 2018

\begin{abstract}
A central factor in the modelling and analysis of the trend is the ability to establish whether a change or trend is present in the climatological record and to quantify this trend if it is present. The trend in a time series data can be expressed by a suitable linear (parametric) or nonlinear (non-parametric) model depending on the behaviour of the available data. The aim of this research is to detect and estimate the magnitude of trend associated with rainfall data from Warri and Benin City which are located within the coastal region of Nigeria using non-parametric Mann-Kendall test statistical approach. Monthly data for thirty six (36) years spanning from 1980 to 2016 was used as input parameters for the analysis. Infilling of the missing records was done with the aid of expectation maximization algorithm. Preprocessing of the rainfall data was done by conducting numerous time series validation test such as test of homogeneity, test of normality and outlier detection. Homogeneity test was aimed at testing the assumption of same population distribution; outlier detection was to detect the presence of bias in the data while test of normality was done to validate the claim that climatic data are not always normally distributed. In addition to testing the normality assumption of the data, normality test was also employed to select the most suitable trend detection and estimation technique. Results of the analysis revealed that the rainfall data from Warri and Benin City are statistically homogeneous. The records did not contain outliers and they are not normally distributed as expected for most climatic variables. The non-parametric trend detection and estimation analysis revealed that the rainfall data from Benin City shows statistical significant evidence of an increasing trend with a computed $\mathrm{M}-\mathrm{K}$ trend value of +124 . Although, the rainfall records from Warri do not have sufficient statistical evidence of a significant trend, the computed M-K trend value was - 96 which is; evidence of a decreasing trend.
\end{abstract}

Keywords: Expectation Maximization Algorithm, Normality Test, Outlier Detection, Mann-Kendall Test, Non-parametric Analysis

\section{Introduction}

Trend in rainfall data have a great impact on the hydrological cycle and thus involve both the character and quantity of water resources. Analysis of trend in rainfall data also aids to see the result of rainfall variability on the occurrence of drought and flood [3]. Although numerous variables such as temperature, vegetation affects the hydrological cycle, precipitation remains the key climatic variable that governs the hydrologic cycle and the availability of water resources. Numerous studies have analyzed the changes in precipitation patterns in global as well as regional scale. Recent studies have also suggested that analysis of hydro-climatic variables should be done at the local scale rather than at a large or global scale because the trends and their impacts may be different from one location to the other [6].

There are many different ways in which changes in hydrometeorological series can take place. A change can occur abruptly (step change) or gradually (trend) or may take more complex forms. A time series is pronounced to have trends, if there is a significant correlation (positive or minus) between the observations and time. Trends and shifts in hydrologic time series are usually introduced due to natural or artificial 
changes. Natural changes in hydrologic variables are usually gradual and are caused by a global or regional climate change, which would be a representative of changes occurring over the study area [7], [8]. Changes in monitoring variables that may not be able to be extrapolated over a study area could be caused by a gradual urbanization of the area surrounding the monitoring site, changes in the method of measurement at the monitoring site, or by moving the monitoring site even a short distance away. The artificial change is usually mentioned in the overall record at a monitoring site, but this information is not always shown in the sites' data series. Thus, variables that appear to have a trend may actually just represent a change in climatological conditions near the monitoring site. In such a case, the affected climatological data should be changed so that the values are better represented of the study area as a whole [2] [4].

A central factor in the modeling and analysis of the trend is the ability to establish whether a change or trend is present in the climatological record and to quantify this trend, if it is present. The trend in a time series data can be expressed by a suitable linear (parametric) or nonlinear (non-parametric) model depending on the behaviour of the available data. The linear model is widely used in hydrology and the simplest of the linear models normally employed for trend detection is the Student's t-test which requires that the series under testing should be normally distributed [2]. Most importantly therefore, whether or not the sample data follow a normal distribution has to be examined prior to the analysis in order to choose the appropriate model for trend detection and analysis. Unfortunately, most researchers ignore this important check. If normality is violated (if the available data do not follow a normal distribution), then, the nonparametric test such as the Mann-Kendall Test is commonly applied to assess the statistical significance of trends [10], [11], [9].

\section{Method}

\subsection{Description of Study Area}

Nigeria is located in West Africa between latitude $4^{0} \mathrm{~N}$ and $14^{0} \mathrm{~N}$ and between longitudes $2^{0} \mathrm{E}$ and $15^{\circ} \mathrm{E}$. It has a total area of $925,796 \mathrm{~km}^{2}$. The ecological zones of the country are broadly grouped into three, which are; Sahel, Savannah and the Guinea zones. Nigeria is affected by the Tropical Continental and Tropical Maritime air masses. The Tropical Continental is responsible for the dry season while the Tropical Maritime is responsible for the rainy season. The intervening periods of transition from the real onset and cessation of rain falls between February and April and between September and November respectively. Also a depression is indicated in the rainfall amount during the month of August and this has been named "the little dry season" or "August break" or "midsummer" [1].

The Nigerian coastal zone sprawls a total of eight states, out of the thirty-six states of the Federation, namely: AkwaIbom, Bayelsa, Cross River, Delta, Edo, Lagos, Ondo, and Rivers. The coastal states are estimated to account for $25 \%$ of the national population. The coastal areas stretch inland for a distance of about $15 \mathrm{~km}$ in Lagos in the West to about $150 \mathrm{~km}$ in the Edo/ Delta and about $25 \mathrm{~km}$ east of the Niger River. The coastline stretches for about $853 \mathrm{~km}$ comprising inshore waters, coastal lagoons, estuaries and mangrove especially in the Niger Delta. Figure 1 shows the Nigeria coastal zones.

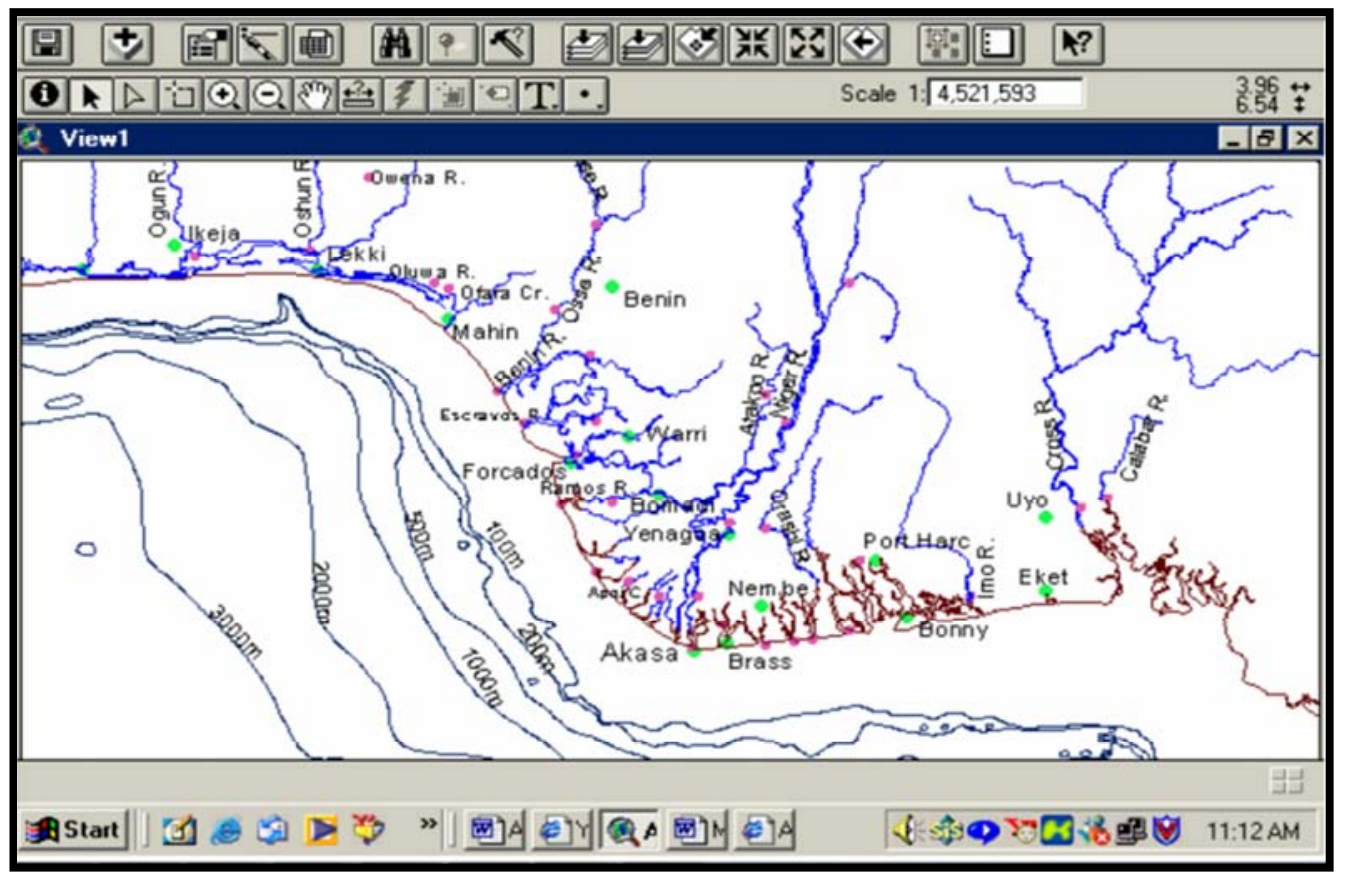

Figure 1. Nigeria's Coastal Zones.

The areas chosen as case study for this research are Benin City the capital of Edo State and Warri the economic hub of
Delta State. Benin City is located within the rainforest region with mean annual rainfall of $2100 \mathrm{~mm}$ and mean monthly 
temperature ranging from a minimum of $23^{\circ} \mathrm{C}$ to a maximum of $27^{\circ} \mathrm{C}$. The city lies on a gently sloping coastal plain in a drainage divide between the head waters of the sub catchment system of Ikpoba and Ogba rivers.

Warri is the economic nerve center of Delta state and also the most populated town. It is located in the southern end of the state. The state lies approximately between Longitude $5^{\circ}$ 00 and $60.45^{\prime}$ East and Latitude $5^{\circ} 00$ and $6^{\circ} .30^{\prime}$ North. It is bounded in the north and west by Edo State, the east by Anambra, Imo, and Rivers States, southeast by Bayelsa State, and on the southern flank is the Bight of Benin which covers about 160 kilometres of the state's coastline.

\subsection{Data Collection}

The rainfall data needed for this study was collected from Nigerian Meteorological Center, Oshodi; Lagos State, Nigeria. The data includes monthly precipitation data for 36 years spanning between; 1980 to 2016 . Table 1 shows the records of the data from the different states under study

Table 1. Rainfall records for the states under study.

\begin{tabular}{llll}
\hline S/no & Study Area & Rainfall Records & No of missing records \\
\hline 1 & Warri & 432 & 25 \\
2 & Benin City & 432 & 27 \\
\hline
\end{tabular}

\subsection{Preprocessing of Data}

Climatic data collected was processed and used on monthly and seasonal basis for analytical convenience. Preprocessing of the data was aimed at:
i. Analysis of missing values
ii. Detection of outliers
iii. Test of Homogeneity and
iv. Test of normality

\subsection{Trend Detection Using Mann-Kendall Test (M-K Test)}

The test is usually known as Kendall tau statistics and has been widely used to test for randomness against trend in hydrology and climatology. It was employed in this study because it is a non-parametric ranked based procedure which is robust to the influence of extremes and good for use with skewed variables [2]. In addition, the test is highly resistant to the effects of outliers. The test was used to assess the presence of trends that is associated with the time series data.

\section{Results and Discussion}

The rainfall data collected from Warri and Benin City showing the missing values is presented in Figure 2 and Figure 3 .

\begin{tabular}{|c|c|c|c|c|c|c|c|c|c|c|c|c|}
\hline Year & January & February & March & April & May & June & July & August & September & October & November & Decmber \\
\hline 1981 & 41.9 & 56.4 & 68.3 & 83.3 & 334.6 & 296.2 & 362.3 & 348.0 & 410.7 & 269.3 & 76.7 & 2.2 \\
\hline 1982 & & 203.5 & 567.8 & 347.3 & 277.9 & 388.3 & 409.3 & 376.3 & 292.6 & 110.7 & 55.2 & 28.0 \\
\hline 1983 & & 49.1 & 6.0 & 128.1 & 351.7 & 406.5 & 351.3 & 103.0 & 670.8 & 177.0 & 87.5 & 190.6 \\
\hline 1984 & & 12.6 & 171.5 & 221.8 & 243.0 & 271.2 & 516.1 & 439.5 & 479.4 & 238.8 & 100.4 & 3.2 \\
\hline 1985 & 35.9 & 60.8 & 175.6 & 147.4 & 207.1 & 594.0 & 461.2 & 649.6 & 431.8 & 139.1 & 62.1 & 12.2 \\
\hline 1986 & 18.4 & 50.0 & 169.9 & 246.8 & 220.1 & 281.8 & 726.4 & 318.6 & 255.7 & 543.4 & 84.7 & \\
\hline 1987 & & 83.7 & 155.4 & 88.9 & 301.2 & 206.0 & 576.5 & 623.8 & 390.1 & 312.1 & 37.9 & \\
\hline 1988 & 18.2 & 108.4 & 121.3 & 185.8 & 177.5 & 349.5 & 313.7 & 255.8 & 634.6 & 295.5 & 87.5 & 161.0 \\
\hline 1989 & & 6.3 & 109.1 & 171.0 & 256.0 & 374.1 & 452.9 & 385.8 & 371.8 & 272.7 & 156.3 & 15.5 \\
\hline 1990 & 77.4 & 7.5 & 38.4 & 222.1 & 137.0 & 467.7 & 582.9 & 733.6 & 386.0 & 318.2 & 99.9 & 28.1 \\
\hline 1991 & & & 112.3 & 459.3 & 219.3 & 230.5 & 599.7 & 497.3 & 359.9 & 353.1 & 80.2 & 9.1 \\
\hline 1992 & & 40.5 & 139.4 & 93.6 & 260.0 & 519.8 & 758.3 & 297.5 & 337.3 & 453.6 & 135.3 & 25.0 \\
\hline 1993 & & 40.5 & 189.0 & 286.1 & 231.2 & 128.7 & 868.4 & 492.7 & 363.1 & 201.3 & 175.4 & 34.2 \\
\hline 1994 & 70.2 & 17.6 & 70.2 & 126.9 & 182.9 & 362.0 & 453.7 & 398.5 & 616.9 & 455.3 & 52.8 & \\
\hline 1995 & & 43.0 & 298.1 & 188.4 & 264.8 & 393.8 & 398.7 & 533.1 & 607.6 & 396.5 & 171.6 & 42.2 \\
\hline 1996 & 11.2 & 134.4 & 244.5 & 337.3 & 278.9 & 304.0 & 167.3 & 383.2 & 520.7 & 292.9 & 31.3 & \\
\hline 1997 & 28.9 & & 131.2 & 279.1 & 300.8 & 295.5 & 666.2 & 385.8 & 331.5 & 611.6 & 180.7 & 16.0 \\
\hline 1998 & 19.3 & 74.4 & 69.7 & 170.6 & 227.2 & 352.0 & 562.1 & 76.9 & 575.7 & 197.2 & 118.5 & 4.9 \\
\hline 1999 & 51.3 & 71.2 & 154.0 & 186.0 & 316.4 & 168.3 & 644.4 & 229.8 & 501.0 & 652.0 & 419.6 & 2.6 \\
\hline 2000 & 18.3 & 41.9 & 132.1 & 239.6 & 312.9 & 404.4 & 261.0 & 471.7 & 388.5 & 304.2 & 108.5 & 39.1 \\
\hline 2001 & 4.2 & 6.1 & 129.0 & 282.1 & 334.2 & 295.1 & 340.2 & 342.3 & 354.2 & 245.0 & 42.9 & 14.9 \\
\hline 2002 & & 65.8 & 83.0 & 309.8 & 232.9 & 375.9 & 628.0 & 609.7 & 517.0 & 454.0 & 36.1 & \\
\hline 2003 & 53.8 & 6.0 & 153.3 & 238.6 & 168.3 & 234.4 & 243.2 & 306.3 & 436.1 & 364.5 & 115.0 & 9.9 \\
\hline 2004 & 25.4 & 67.0 & 46.3 & 159.4 & 379.1 & 243.6 & 795.7 & 454.7 & 497.2 & 334.1 & 48.0 & 13.5 \\
\hline 2005 & 37.3 & 21.7 & 191.2 & 104.1 & 315.1 & 216.4 & 592.4 & 146.7 & 432.5 & 203.6 & 68.6 & 39.0 \\
\hline 2006 & 111.0 & 171.9 & 177.6 & 149.2 & 453.9 & 229.4 & 368.4 & 305.6 & 528.2 & 493.2 & 25.0 & 17.7 \\
\hline 2007 & & & 23.5 & 146.3 & 287.1 & 550.3 & 364.2 & 141.4 & 512.8 & 327.7 & 65.3 & 33.5 \\
\hline 2008 & 1.7 & 10.0 & 182.6 & 270.0 & 357.7 & 467.6 & 451.9 & 635.4 & 422.3 & 214.5 & 75.3 & 23.8 \\
\hline 2009 & 13.0 & 83.6 & 71.1 & 139.9 & 210.3 & 575.6 & 418.9 & 416.6 & 424.1 & 341.4 & 64.9 & 15.7 \\
\hline 2010 & 5.4 & 119.4 & 89.5 & 218.9 & 380.0 & 268.7 & 191.8 & 302.2 & 546.6 & 296.3 & 157.7 & 4.7 \\
\hline 2011 & & 139.1 & 68.3 & 143.7 & 366.9 & 332.5 & 672.6 & 459.5 & 254.8 & 153.0 & 114.1 & 0.0 \\
\hline 2012 & 18.8 & 168.0 & 47.1 & 137.6 & 333.4 & 469.9 & 677.5 & 132.9 & 333.0 & 339.6 & 112.2 & 0.0 \\
\hline 2013 & 107.1 & 15.7 & 151.6 & 207.3 & 231.3 & 458.6 & 497.5 & 183.8 & 185.2 & 483.6 & 203.1 & 37.3 \\
\hline 2014 & & 54.7 & 132.4 & 207.8 & 225.8 & 412.3 & 467.9 & 176.5 & 165.4 & 345.9 & & 21.6 \\
\hline 2015 & 12.5 & 78.9 & 143.2 & 204.2 & & 367.2 & 478.9 & 174.3 & 143.7 & 315.6 & 87.9 & 15.9 \\
\hline 2016 & & 84.3 & 189.7 & 264.3 & 354.8 & 476.5 & 398.7 & 437.2 & 334.9 & 329.8 & 147.2 & \\
\hline
\end{tabular}

Figure 2. Warri rainfall data showing the missing records. 


\begin{tabular}{|c|c|c|c|c|c|c|c|c|c|c|c|c|}
\hline Year & January & February & March & April & May & June & July & August & September & October & November & Decmber \\
\hline 1981 & . & 6.8 & 108.4 & 119.8 & 277.2 & 180.6 & 276.1 & 230.0 & 394.8 & 178.1 & 12.9 & 1.1 \\
\hline 1982 & 101.9 & 111.4 & 98.2 & 211.4 & 146.0 & 174.1 & 243.9 & 66.8 & 391.0 & 381.9 & 42.4 & \\
\hline 1983 & & 49.4 & 39.0 & 76.8 & 267.4 & 277.2 & 166.5 & 142.8 & 425.2 & 150.3 & 29.8 & 22.8 \\
\hline 1984 & & 45.8 & 87.3 & 59.3 & 120.4 & 134.1 & 223.8 & 181.8 & 235.2 & 154.8 & 4.4 & 2.5 \\
\hline 1985 & 8.7 & 16.0 & 110.5 & 33.2 & 173.4 & 202.5 & 244.3 & 305.2 & 197.9 & 147.5 & 106.1 & \\
\hline 1986 & 5.6 & 45.1 & 109.6 & 52.3 & 162.7 & 65.1 & 214.2 & 117.7 & 222.3 & 166.5 & 67.1 & \\
\hline 1987 & 0.8 & 74.5 & 100.2 & 112.5 & 151.4 & 217.0 & 269.5 & 722.5 & 348.2 & 299.8 & 39.9 & \\
\hline 1988 & 7.0 & 71.1 & 154.8 & 136.5 & 168.0 & 227.0 & 393.0 & 191.2 & 445.4 & 273.2 & 23.3 & 58.6 \\
\hline 1989 & & 25.8 & 66.1 & 152.0 & 140.4 & 343.4 & 279.2 & 427.8 & 157.3 & 365.1 & 14.2 & \\
\hline 1990 & 19.6 & 18.8 & & 256.8 & 181.4 & 204.1 & 353.4 & 614.5 & 296.9 & 288.9 & 33.7 & 168.6 \\
\hline 1991 & & 58.1 & 123.5 & 386.3 & 196.7 & 207.2 & 656.2 & 382.6 & 268.0 & 267.9 & 39.2 & 11.9 \\
\hline 1992 & & 0.2 & 41.4 & 222.7 & 240.0 & 335.8 & 515.9 & 76.4 & 256.3 & 292.2 & 35.6 & \\
\hline 1993 & & 9.6 & 135.0 & 95.4 & 198.2 & 208.8 & 191.4 & 433.9 & 257.6 & 174.2 & 108.1 & 48.6 \\
\hline 1994 & 27.5 & 14.6 & 111.4 & 149.8 & 327.9 & 351.3 & 444.4 & 461.4 & 391.7 & 204.5 & 47.0 & . \\
\hline 1995 & & 50.6 & 165.4 & 217.9 & 226.9 & 286.2 & 383.3 & 580.8 & 383.3 & 240.2 & 124.7 & 9.1 \\
\hline 1996 & 7.0 & 92.6 & 188.2 & 298.3 & 322.2 & 28.1 & 182.3 & 392.3 & 476.0 & 292.7 & 5.6 & 1.1 \\
\hline 1997 & 75.3 & & 104.0 & 230.9 & 305.3 & 203.3 & 285.0 & 160.2 & 222.9 & 338.4 & 176.6 & 98.9 \\
\hline 1998 & 44.1 & 1.8 & 104.6 & 104.8 & 214.6 & 214.4 & 506.1 & 95.6 & 387.9 & 244.0 & 58.8 & 42.0 \\
\hline 1999 & 86.3 & 64.4 & 98.3 & 119.6 & 161.7 & 99.5 & 412.3 & 232.0 & 369.0 & 472.5 & 97.8 & 9.4 \\
\hline 2000 & 4.0 & 73.0 & 60.8 & 170.0 & 191.8 & 413.7 & 294.7 & 237.9 & 345.0 & 351.2 & 49.0 & 48.7 \\
\hline 2001 & 18.8 & 10.1 & 119.3 & 394.3 & 155.7 & 364.3 & 216.0 & 137.4 & 357.1 & 183.0 & 82.6 & 3.9 \\
\hline 2002 & & 115.0 & 163.5 & 278.1 & 199.4 & 344.9 & 411.3 & 491.8 & 294.8 & 149.5 & 101.0 & 9.9 \\
\hline 2003 & 33.9 & 13.7 & 172.0 & 169.8 & 226.5 & 187.6 & 177.1 & 142.1 & 393.3 & 338.1 & 57.3 & 26.3 \\
\hline 2004 & 28.8 & 28.3 & 68.4 & 118.9 & 250.7 & 448.9 & 288.0 & 403.0 & 297.6 & 299.8 & 94.3 & 1.6 \\
\hline 2005 & & 9.8 & 182.2 & 119.6 & 95.5 & 450.2 & 458.8 & 97.0 & 207.6 & 333.2 & 40.0 & 20.1 \\
\hline 2006 & 33.1 & 22.3 & 146.0 & 117.1 & 394.0 & 240.2 & 462.4 & 359.2 & 334.4 & 217.8 & 32.0 & 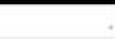 \\
\hline 2007 & & . & 91.6 & 183.5 & 350.2 & 347.9 & 354.3 & 303.3 & 462.5 & 263.5 & 152.0 & 18.5 \\
\hline 2008 & 10.3 & 2.8 & 146.1 & 160.1 & 215.6 & 272.9 & 413.6 & 319.7 & 199.3 & 90.6 & 95.4 & \\
\hline 2009 & 14.6 & 108.6 & 69.8 & 157.8 & 330.5 & 171.8 & 175.0 & 248.3 & 294.5 & 342.9 & 161.0 & 23.0 \\
\hline 2010 & 15.5 & 68.6 & 55.3 & 321.5 & 79.6 & 145.4 & 91.6 & 332.2 & 615.1 & 267.4 & 306.5 & 40.7 \\
\hline 2011 & . & 77.8 & 87.0 & 321.5 & 353.5 & 430.9 & 550.8 & 502.5 & 409.3 & 414.6 & 63.6 & 0.0 \\
\hline 2012 & 47.7 & 53.2 & 74.8 & 157.1 & 383.7 & 490.4 & 395.3 & 124.9 & 255.5 & 283.4 & 186.7 & 0.0 \\
\hline 2013 & 11.9 & 61.8 & 126.2 & 201.0 & 312.2 & 255.6 & 390.4 & 168.1 & 564.0 & 338.8 & 105.8 & 60.1 \\
\hline 2014 & & 54.3 & 125.3 & 204.3 & 332.1 & 245.6 & 456.7 & 156.7 & 523.4 & 336.3 & 103.4 & 42.3 \\
\hline 2015 & 18.7 & 47.9 & 78.6 & 189.6 & 302.4 & & 356.7 & 145.6 & 436.5 & 321.4 & 87.4 & 25.6 \\
\hline 2016 & . & 51.7 & 76.2 & 205.9 & 311.2 & 255.4 & 332.1 & 263.4 & 490.8 & 342.1 & 122.3 & 18.8 \\
\hline
\end{tabular}

Figure 3. Benin City rainfall data showing the missing records.

The missing records were filled using expectation maximization algorithm. The null hypothesis for the little MCAR (missing completely at random) test was formulated as;

$\mathrm{H} 0$; the data are missing completely at random

$\mathrm{H} 1$; data are not missing completely at random

The analysis was conducted at 0.05 degree of freedom that is; $95 \%$ confidence interval. Infilling of the missing records were based on the outcome of the correlation statistics which is critical to the fundamental assumptions of missing value analysis using expectation maximization algorithm. Results of the correlation statistics is presented in Figure 4 and Figure 5

\begin{tabular}{|c|c|c|c|c|c|c|c|c|c|c|c|c|c|}
\hline \multicolumn{14}{|c|}{ EM Correlationss } \\
\hline & 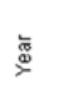 & 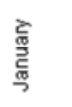 & 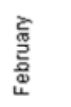 & $\begin{array}{l}\frac{5}{\underline{\omega}} \\
\frac{\mathrm{c}}{2}\end{array}$ & 言 & 商 & 营 & 今े & $\begin{array}{l}\text { 㤎 } \\
\text { 丞 }\end{array}$ & 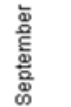 & 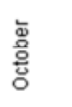 & 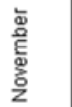 & 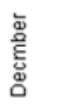 \\
\hline Year & 1 & & & & & & & & & & & & \\
\hline $\begin{array}{l}\text { January } \\
\text { February }\end{array}$ & $\begin{array}{l}.104 \\
142\end{array}$ & $\begin{array}{r}1 \\
-095\end{array}$ & 1 & & & & & & & & & & \\
\hline March & -.211 & .030 & .296 & 1 & & & & & & & & & \\
\hline April & .016 & .452 & .003 & .398 & 1 & & & & & & & & \\
\hline May & .347 & -.064 & .401 & -.003 & -174 & 1 & & & & & & & \\
\hline June & .172 & .040 & .019 & -.111 & -199 & -199 & 1 & & & & & & \\
\hline July & -.050 & .013 & -.112 & -.132 & -.069 & -.078 & -187 & 1 & & & & & \\
\hline August & -.274 & .046 & -.190 & .149 & .279 & -.185 & .044 & .125 & 1 & & & & \\
\hline September & -.312 & -.004 & .016 & -.190 & -.193 & .001 & -.104 & -316 & .012 & 1 & & & \\
\hline October & .168 & .439 & -100 & -.148 & .023 & -.054 & -.150 & .218 & -100 & -.041 & 1 & & \\
\hline November & .128 & .128 & -.146 & .011 & .007 & .020 & -.168 & .219 & .206 & -.099 & .434 & 1 & \\
\hline Decmber & -.270 & -158 & .027 & .092 & .083 & .111 & .113 & .261 & -.251 & .440 & -148 & .098 & 1 \\
\hline
\end{tabular}

Figure 4. EM correlation statistics for Warri rainfall data. 


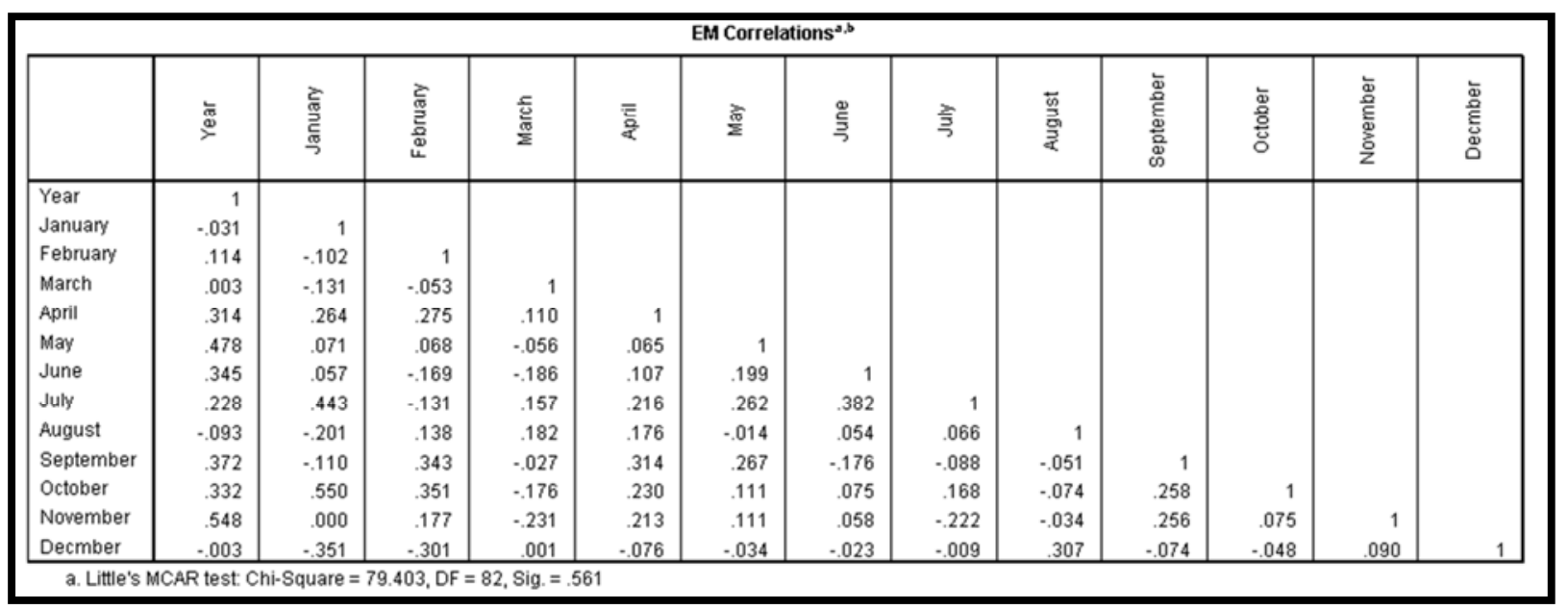

Figure 5. EM correlation statistics for Benin City rainfall data.

From the correlation statistics of Figure 4 and Figure 5, a Probability $(\mathrm{P})$ value of 0.833 and 0.561 were observed. Since P $>0.05$ we accepted the null hypothesis H0 and concluded that the data were missing completely at random. Based on this conclusion EMA was then employed to fill the missing values. The complete rainfall records for Warri and Benin City is presented in Figure 6 and Figure 7.

To detect the presence of outlier in the rainfall data, the labeling rule method was employed. The labeling rule is the statistical method of detecting the presence of outliers in data sets using the 25th percentile (lower bound) and the 75th percentile (upper bound). The underlying mathematical equation based on the lower and the upper bound is presented as follows:

$$
\begin{aligned}
& \text { Lower Bound } \mathrm{Q}_{1}-\left(2.2 \times\left(Q_{3}-Q_{1}\right)\right. \\
& \text { Upper Bound } \mathrm{Q}_{3}+\left(2.2 \times\left(Q_{3}-Q_{1}\right)\right.
\end{aligned}
$$

At 0.05 degree of freedom, any data lower than $\mathrm{Q}_{1}$ or greater than $\mathrm{Q}_{3}$ was considered an outlier [4]. The descriptive statistics of the data based on the outlier detection analysis is

\begin{tabular}{|c|c|c|c|c|c|c|c|c|c|c|c|c|}
\hline Year & January & February & March & April & May & June & July & August & September & October & November & Decmber \\
\hline 1981 & 41.9 & 56.4 & 68.3 & 83.3 & 334.6 & 296.2 & 362.3 & 348.0 & 410.7 & 269.3 & 76.7 & 2.2 \\
\hline 1962 & 10.2 & 203.5 & 567.8 & 347.3 & 277.9 & 368.3 & 409.3 & 376.3 & 292.6 & 110.7 & 55.2 & 28.0 \\
\hline 1983 & 20.8 & 49.1 & 6.0 & 128.1 & 351.7 & 406.5 & 351.3 & 103.0 & 670.8 & 177.0 & 87.5 & 190.6 \\
\hline 1984 & 16.5 & 12.6 & 171.5 & 221.8 & 243.0 & 271.2 & 516.1 & 439.5 & 479.4 & 238.8 & 100.4 & 3.2 \\
\hline 1985 & 35.9 & 60.8 & 175.6 & 147.4 & 207.1 & 594.0 & 461.2 & 649.6 & 431.8 & 139.1 & 62.1 & 12.2 \\
\hline 1986 & 18.4 & 50.0 & 169.9 & 246.8 & 220.1 & 281.8 & 726.4 & 318.6 & 255.7 & 543.4 & 84.7 & 16.7 \\
\hline 1987 & 79.4 & 83.7 & 155.4 & 88.9 & 301.2 & 206.0 & 576.5 & 623.8 & 390.1 & 312.1 & 37.9 & 11.2 \\
\hline 1988 & 18.2 & 108.4 & 121.3 & 185.8 & 177.5 & 349.5 & 313.7 & 255.8 & 634.6 & 295.5 & 87.5 & 161.0 \\
\hline 1969 & 26.4 & 6.3 & 109.1 & 171.0 & 256.0 & 374.1 & 452.9 & 385.8 & 371.8 & 272.7 & 156.3 & 15.5 \\
\hline 1990 & 77.4 & 7.5 & 38.4 & 222.1 & 137.0 & 467.7 & 582.9 & 733.6 & 386.0 & 318.2 & 99.9 & 28.1 \\
\hline 1991 & 40.1 & 34.5 & 112.3 & 459.3 & 219.3 & 230.5 & 599.7 & 497.3 & 359.9 & 353.1 & 80.2 & 9.1 \\
\hline 1992 & 61.5 & 40.5 & 139.4 & 93.6 & 260.0 & 519.8 & 758.3 & 297.5 & 337.3 & 453.6 & 135.3 & 25.0 \\
\hline 1993 & 7.0 & 40.5 & 189.0 & 286.1 & 231.2 & 128.7 & 868.4 & 492.7 & 363.1 & 201.3 & 175.4 & 34.2 \\
\hline 1994 & 70.2 & 17.6 & 70.2 & 126.9 & 182.9 & 362.0 & 453.7 & 398.5 & 616.9 & 455.3 & 52.8 & 49.9 \\
\hline 1995 & 69.5 & 43.0 & 298.1 & 188.4 & 264.8 & 393.8 & 398.7 & 533.1 & 607.6 & 396.5 & 171.6 & 42.2 \\
\hline 1996 & 11.2 & 134.4 & 244.5 & 337.3 & 278.9 & 304.0 & 167.3 & 383.2 & 520.7 & 292.9 & 31.3 & 46.9 \\
\hline 1997 & 28.9 & 59.7 & 131.2 & 279.1 & 300.8 & 295.5 & 666.2 & 385.8 & 331.5 & 611.6 & 180.7 & 16.0 \\
\hline 1998 & 19.3 & 74.4 & 69.7 & 170.6 & 227.2 & 352.0 & 562.1 & 76.9 & 575.7 & 197.2 & 118.5 & 4.9 \\
\hline 1999 & 51.3 & 71.2 & 154.0 & 186.0 & 316.4 & 168.3 & 644.4 & 229.8 & 501.0 & 652.0 & 419.6 & 2.6 \\
\hline 2000 & 18.3 & 41.9 & 132.1 & 239.6 & 312.9 & 404.4 & 261.0 & 471.7 & 388.5 & 304.2 & 108.5 & 39.1 \\
\hline 2001 & 4.2 & 6.1 & 129.0 & 282.1 & 334.2 & 295.1 & 340.2 & 342.3 & 354.2 & 245.0 & 42.9 & 14.9 \\
\hline 2002 & 24.2 & 65.8 & 83.0 & 309.8 & 232.9 & 375.9 & 628.0 & 609.7 & 517.0 & 454.0 & 36.1 & 19.2 \\
\hline 2003 & 53.8 & 6.0 & 153.3 & 238.6 & 168.3 & 234.4 & 243.2 & 306.3 & 436.1 & 364.5 & 115.0 & 9.9 \\
\hline 2004 & 25.4 & 67.0 & 46.3 & 159.4 & 379.1 & 243.6 & 795.7 & 454.7 & 497.2 & 334.1 & 48.0 & 13.5 \\
\hline 2005 & 37.3 & 21.7 & 191.2 & 104.1 & 315.1 & 216.4 & 592.4 & 146.7 & 432.5 & 203.6 & 68.6 & 39.0 \\
\hline 2006 & 111.0 & 171.9 & 177.6 & 149.2 & 453.9 & 229.4 & 368.4 & 305.6 & 528.2 & 493.2 & 25.0 & 17.7 \\
\hline 2007 & 23.1 & 85.1 & 23.5 & 146.3 & 287.1 & 550.3 & 364.2 & 141.4 & 512.8 & 327.7 & 65.3 & 33.5 \\
\hline 2008 & 1.7 & 10.0 & 182.6 & 270.0 & 357.7 & 467.6 & 451.9 & 635.4 & 422.3 & 214.5 & 75.3 & 23.8 \\
\hline 2009 & 13.0 & 83.6 & 71.1 & 139.9 & 210.3 & 575.6 & 418.9 & 416.6 & 424.1 & 341.4 & 64.9 & 15.7 \\
\hline 2010 & 5.4 & 119.4 & 89.5 & 218.9 & 380.0 & 268.7 & 191.8 & 302.2 & 546.6 & 296.3 & 157.7 & 4.7 \\
\hline 2011 & 16.3 & 139.1 & 68.3 & 143.7 & 366.9 & 332.5 & 672.6 & 459.5 & 254.8 & 153.0 & 114.1 & 0.0 \\
\hline 2012 & 18.8 & 168.0 & 47.1 & 137.6 & 333.4 & 469.9 & 677.5 & 132.9 & 333.0 & 339.6 & 112.2 & 0.0 \\
\hline 2013 & 107.1 & 15.7 & 151.6 & 207.3 & 231.3 & 458.6 & 497.5 & 183.8 & 185.2 & 483.6 & 203.1 & 37.3 \\
\hline 2014 & 40.8 & 54.7 & 132.4 & 207.8 & 225.8 & 412.3 & 467.9 & 176.5 & 165.4 & 345.9 & 130.8 & 21.6 \\
\hline 2015 & 12.5 & 78.9 & 143.2 & 204.2 & 335.4 & 367.2 & 478.9 & 174.3 & 143.7 & 315.6 & 87.9 & 15.9 \\
\hline 2016 & 24.2 & 84.3 & 189.7 & 264.3 & 354.8 & 476.5 & 398.7 & 437.2 & 334.9 & 329.8 & 147.2 & 3.5 \\
\hline
\end{tabular}
presented in Figure 8 and Figure 9

Figure 6 Complete rainfall records for Warri using expectation maximization algorithm. 


\begin{tabular}{|c|c|c|c|c|c|c|c|c|c|c|c|c|}
\hline Year & January & February & March & April & May & June & July & August & September & October & November & Decmber \\
\hline 1981 & 40.8 & 6.8 & 108.4 & 119.8 & 277.2 & 180.6 & 276.1 & 230.0 & 394.8 & 178.1 & 12.9 & 1.1 \\
\hline 1982 & 101.9 & 111.4 & 98.2 & 211.4 & 146.0 & 174.1 & 243.9 & 66.8 & 391.0 & 381.9 & 42.4 & 183.0 \\
\hline 1983 & 16.1 & 49.4 & 39.0 & 76.8 & 267.4 & 277.2 & 166.5 & 142.8 & 425.2 & 150.3 & 29.8 & 22.8 \\
\hline 1984 & 7.2 & 45.8 & 87.3 & 59.3 & 120.4 & 134.1 & 223.8 & 181.8 & 235.2 & 154.8 & 4.4 & 2.5 \\
\hline 1985 & 8.7 & 16.0 & 110.5 & 33.2 & 173.4 & 202.5 & 244.3 & 305.2 & 197.9 & 147.5 & 106.1 & 81.3 \\
\hline 1986 & 5.6 & 45.1 & 109.6 & 52.3 & 162.7 & 65.1 & 214.2 & 117.7 & 222.3 & 166.5 & 67.1 & 32.6 \\
\hline 1987 & 0.8 & 74.5 & 100.2 & 112.5 & 151.4 & 217.0 & 269.5 & 722.5 & 348.2 & 299.8 & 39.9 & 73.4 \\
\hline 1988 & 7.0 & 71.1 & 154.8 & 136.5 & 168.0 & 227.0 & 393.0 & 191.2 & 445.4 & 273.2 & 23.3 & 58.6 \\
\hline 1989 & 48.3 & 25.8 & 66.1 & 152.0 & 140.4 & 343.4 & 279.2 & 427.8 & 157.3 & 365.1 & 14.2 & 53.0 \\
\hline 1990 & 19.6 & 18.8 & 126.9 & 256.8 & 181.4 & 204.1 & 353.4 & 614.5 & 296.9 & 288.9 & 33.7 & 168.6 \\
\hline 1991 & 86.5 & 58.1 & 123.5 & 386.3 & 196.7 & 207.2 & 656.2 & 382.6 & 268.0 & 267.9 & 39.2 & 11.9 \\
\hline 1992 & 87.1 & 0.2 & 41.4 & 222.7 & 240.0 & 335.8 & 515.9 & 76.4 & 256.3 & 292.2 & 35.6 & 21.4 \\
\hline 1993 & 11.6 & 9.6 & 135.0 & 95.4 & 198.2 & 208.8 & 191.4 & 433.9 & 257.6 & 174.2 & 108.1 & 48.6 \\
\hline 1994 & 27.5 & 14.6 & 111.4 & 149.8 & 327.9 & 351.3 & 444.4 & 461.4 & 391.7 & 204.5 & 47.0 & 52.3 \\
\hline 1995 & 44.5 & 50.6 & 165.4 & 217.9 & 226.9 & 286.2 & 383.3 & 580.8 & 383.3 & 240.2 & 124.7 & 9.1 \\
\hline 1996 & 7.0 & 92.6 & 188.2 & 298.3 & 322.2 & 28.1 & 182.3 & 392.3 & 476.0 & 292.7 & 5.6 & 1.1 \\
\hline 1997 & 75.3 & 33.8 & 104.0 & 230.9 & 305.3 & 203.3 & 285.0 & 160.2 & 222.9 & 338.4 & 176.6 & 98.9 \\
\hline 1998 & 44.1 & 1.8 & 104.6 & 104.8 & 214.6 & 214.4 & 506.1 & 95.6 & 387.9 & 244.0 & 58.8 & 42.0 \\
\hline 1999 & 86.3 & 64.4 & 98.3 & 119.6 & 161.7 & 99.5 & 412.3 & 232.0 & 369.0 & 472.5 & 97.8 & 9.4 \\
\hline 2000 & 4.0 & 73.0 & 60.8 & 170.0 & 191.8 & 413.7 & 294.7 & 237.9 & 345.0 & 351.2 & 49.0 & 48.7 \\
\hline 2001 & 18.8 & 10.1 & 119.3 & 394.3 & 155.7 & 364.3 & 216.0 & 137.4 & 357.1 & 183.0 & 82.6 & 3.9 \\
\hline 2002 & 25.5 & 115.0 & 163.5 & 278.1 & 199.4 & 344.9 & 411.3 & 491.8 & 294.8 & 149.5 & 101.0 & 9.9 \\
\hline 2003 & 33.9 & 13.7 & 172.0 & 169.8 & 226.5 & 187.6 & 177.1 & 142.1 & 393.3 & 338.1 & 57.3 & 26.3 \\
\hline 2004 & 28.8 & 28.3 & 68.4 & 118.9 & 250.7 & 448.9 & 288.0 & 403.0 & 297.6 & 299.8 & 94.3 & 1.6 \\
\hline 2005 & 37.8 & 9.8 & 182.2 & 119.6 & 95.5 & 450.2 & 458.8 & 97.0 & 207.6 & 333.2 & 40.0 & 20.1 \\
\hline 2006 & 33.1 & 22.3 & 146.0 & 117.1 & 394.0 & 240.2 & 462.4 & 359.2 & 334.4 & 217.8 & 32.0 & 18.1 \\
\hline 2007 & 28.3 & 54.2 & 91.6 & 183.5 & 350.2 & 347.9 & 354.3 & 303.3 & 462.5 & 263.5 & 152.0 & 18.5 \\
\hline 2008 & 10.3 & 2.8 & 146.1 & 160.1 & 215.6 & 272.9 & 413.6 & 319.7 & 199.3 & 90.6 & 95.4 & 19.9 \\
\hline 2009 & 14.6 & 108.6 & 69.8 & 157.8 & 330.5 & 171.8 & 175.0 & 248.3 & 294.5 & 342.9 & 161.0 & 23.0 \\
\hline 2010 & 15.5 & 68.6 & 55.3 & 321.5 & 79.6 & 145.4 & 91.6 & 332.2 & 615.1 & 267.4 & 306.5 & 40.7 \\
\hline 2011 & 64.8 & 77.8 & 87.0 & 321.5 & 353.5 & 430.9 & 550.8 & 502.5 & 409.3 & 414.6 & 63.6 & 0.0 \\
\hline 2012 & 47.7 & 53.2 & 74.8 & 157.1 & 383.7 & 490.4 & 395.3 & 124.9 & 255.5 & 283.4 & 186.7 & 0.0 \\
\hline 2013 & 11.9 & 61.8 & 126.2 & 201.0 & 312.2 & 255.6 & 390.4 & 168.1 & 564.0 & 338.8 & 105.8 & 60.1 \\
\hline 2014 & 28.7 & 54.3 & 125.3 & 204.3 & 332.1 & 245.6 & 456.7 & 156.7 & 523.4 & 336.3 & 103.4 & 42.3 \\
\hline 2015 & 18.7 & 47.9 & 78.6 & 189.6 & 302.4 & 225.2 & 356.7 & 145.6 & 436.5 & 321.4 & 87.4 & 25.6 \\
\hline 2016 & 25.6 & 51.7 & 76.2 & 205.9 & 311.2 & 255.4 & 332.1 & 263.4 & 490.8 & 342.1 & 122.3 & 18.8 \\
\hline
\end{tabular}

Figure 7. Complete rainfall records for Benin City using expectation maximization algorithm.

\begin{tabular}{|c|c|c|c|c|c|c|c|c|}
\hline \multicolumn{6}{|c|}{ Descriptives } & & & \\
\hline & & & & Statistic & Std. Error & & & \\
\hline \multirow[t]{13}{*}{ Rainfall } & Mean & \multirow{13}{*}{\multicolumn{2}{|c|}{$\begin{array}{l}\text { Lower Bound } \\
\text { Upper Bound }\end{array}$}} & 234.880 & 8.9107 & & & \\
\hline & 95\% Confidence Interval & & & 217.366 & & & & \\
\hline & & & & 252.394 & & & & \\
\hline & $5 \%$ Trimmed Mean & & & 223.538 & & & & \\
\hline & Median & & & 199.250 & & & & \\
\hline & Variance & & & $3.430 \mathrm{E} 4$ & & & & \\
\hline & Std. Deviation & & & 1.8521E2 & & & & \\
\hline & Minimum & & & & & & & \\
\hline & Maximum & & & 868.4 & & & & \\
\hline & Range & & & 868.4 & & & & \\
\hline & Interquartile Range & & & 289.2 & & & & \\
\hline & Skewness & & & .708 & .117 & & & \\
\hline & Kurtosis & & & -.190 & .234 & & & \\
\hline \multicolumn{9}{|c|}{ Percentiles } \\
\hline & & \multicolumn{7}{|c|}{ Percentiles } \\
\hline & & 5 & 10 & 25 & 50 & 75 & 90 & 95 \\
\hline \multicolumn{2}{|c|}{$\begin{array}{l}\text { Weighted Average } \\
\text { (Definition 1) }\end{array}$} & 10.850 & 18.920 & 70.200 & 199.250 & 359.350 & 496.000 & 595.995 \\
\hline Tukey's : & Rainfall & & & 70.200 & 199.250 & 358.800 & & \\
\hline
\end{tabular}

Figure 8. Descriptive statistics of rainfall data from Warri. 


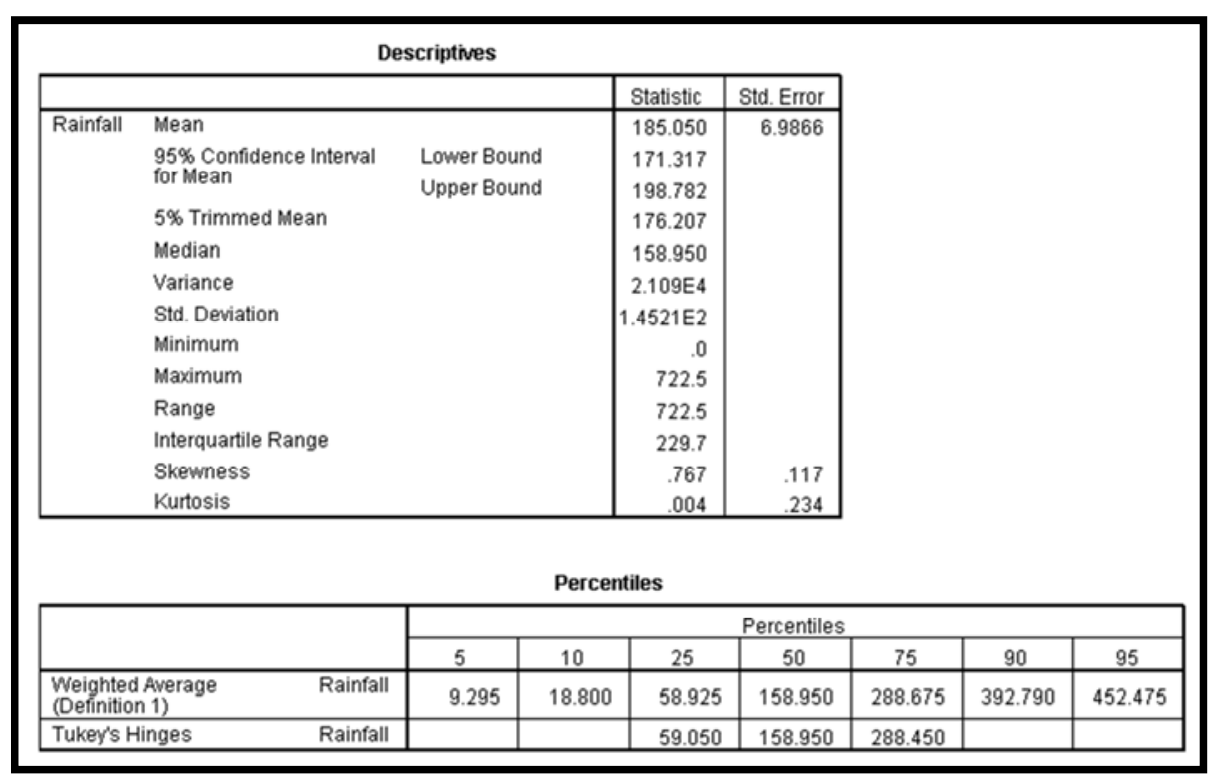

Figure 9. Descriptive statistics of rainfall data from Benin City.

From the result of Figure 8, it was observed that the mean rainfall within the period under study was 234.880 while the variance and standard deviation of the rainfall data were observed to be $3.430 \mathrm{E} 4$ and $1.8521 \mathrm{E} 2$ respectively. Using the weighted average definition, the 25 th percentile $\left(Q_{1}\right)$ was observed to be 70.200 while the 75 th percentile $\left(Q_{3}\right)$ was observed to be 359.350. Adopting the labeling rule of equation (3.1) and (3.2), the lower and upper bound statistics were calculated as follows:

Lower bound $=70.200-(2.2(359.350-70.200))=-565.93$

Upper bound $=359.350+(2.2(359.350-70.200))=995.48$

From the result of Figure 9, it was observed that the mean rainfall within the period under study was 185.050 while the variance and standard deviation of the rainfall data were observed to be $2.109 \mathrm{E} 4$ and $1.452 \mathrm{E} 2$ respectively. Using the weighted average definition, the 25th percentile $\left(Q_{1}\right)$ was observed to be 58.925 while the 75 th percentile $\left(\mathrm{Q}_{3}\right)$ was observed to be 288.675. Adopting the labeling rule of equation (3.1) and (3.2), the lower and upper bound statistics were calculated as follows:

Lower bound $=58.925-(2.2(288.675-58.925))=-446.525$

Upper bound $=288.675+(2.2(288.675-58.925))=794.125$

The extreme value statistics of Warri and Benin City rainfall data which shows the highest and lowest case numbers is presented in Figure 10 and Figure 11

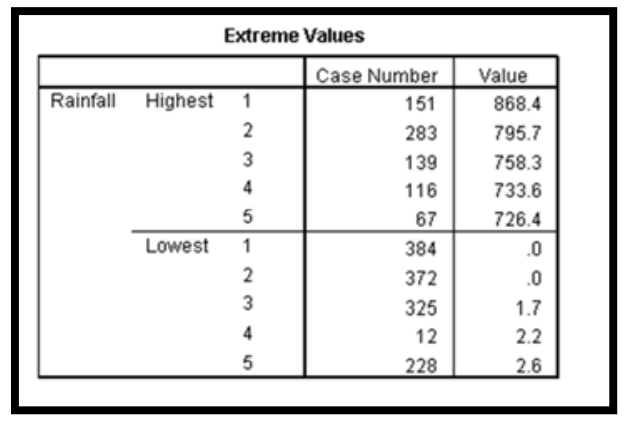

Figure 10. Extreme value statistics of Warri rainfall data.

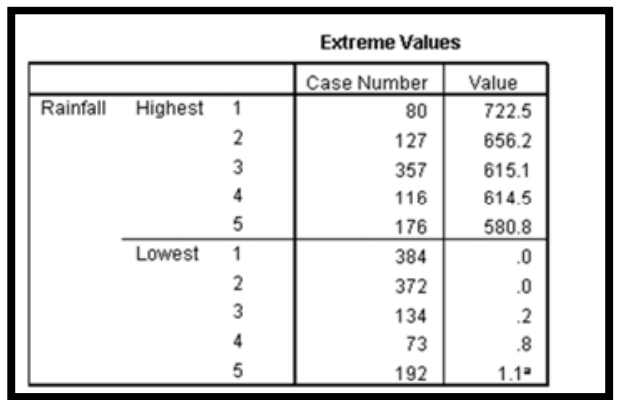

Figure 11. Extreme value statistics of Benin City rainfall data.

From the result of Figure 10, it was observed that the highest rainfall value is $868.4 \mathrm{~mm}$ which is less than the calculated upper bound of $995.48 \mathrm{~mm}$. The lowest rainfall value was observed to be 0.00 which is greater than the calculated lower bound of $-565.93 \mathrm{~mm}$. Since no rainfall value is greater than the calculated upper bound or lower than the calculated lower bound, it was concluded that the rainfall data from Warri is devoid of possible outliers.

From the result of Figure 11, it was observed that the highest rainfall value is $722.5 \mathrm{~mm}$ which is less than the calculated upper bound of $794.125 \mathrm{~mm}$. The lowest rainfall value was observed to be 0.00 which is greater than the calculated lower bound of $-446.525 \mathrm{~mm}$. Since no rainfall value is greater than the calculated upper bound or lower than the calculated lower bound, it was concluded that the rainfall data from Benin City is devoid of possible outliers.

To ascertain that the rainfall data are from the same population distribution, homogeneity test was conducted. Homogeneity test is based on the cumulative deviation from the mean as expressed using the mathematical equation proposed by [5].

$$
S_{k}=\sum_{i=1}^{k}\left(X_{i}-\bar{X}\right) \mathrm{k}=1,-\mathrm{n}
$$


where

$X_{i}=$ The record for the series $\mathrm{X}_{1} \mathrm{X}_{2}$----------- $\mathrm{X}_{\mathrm{n}}$

$X=$ The mean

$\mathrm{S}_{\mathrm{ks}}=$ the residual mass curve

For a homogeneous record, one may expect that the $\mathrm{S}_{\mathrm{ks}}$ fluctuate around zero in the residual mass curve since there is no systematic pattern in the deviation $\mathrm{X}_{\mathrm{i}}$ 's from the average values $X$. To perform the homogeneity test, maximum rainfall values were extracted from the monthly rainfall data and analyzed using the Rainbow software. Results of the homogeneity test is presented in Figure 12 and Figure 13

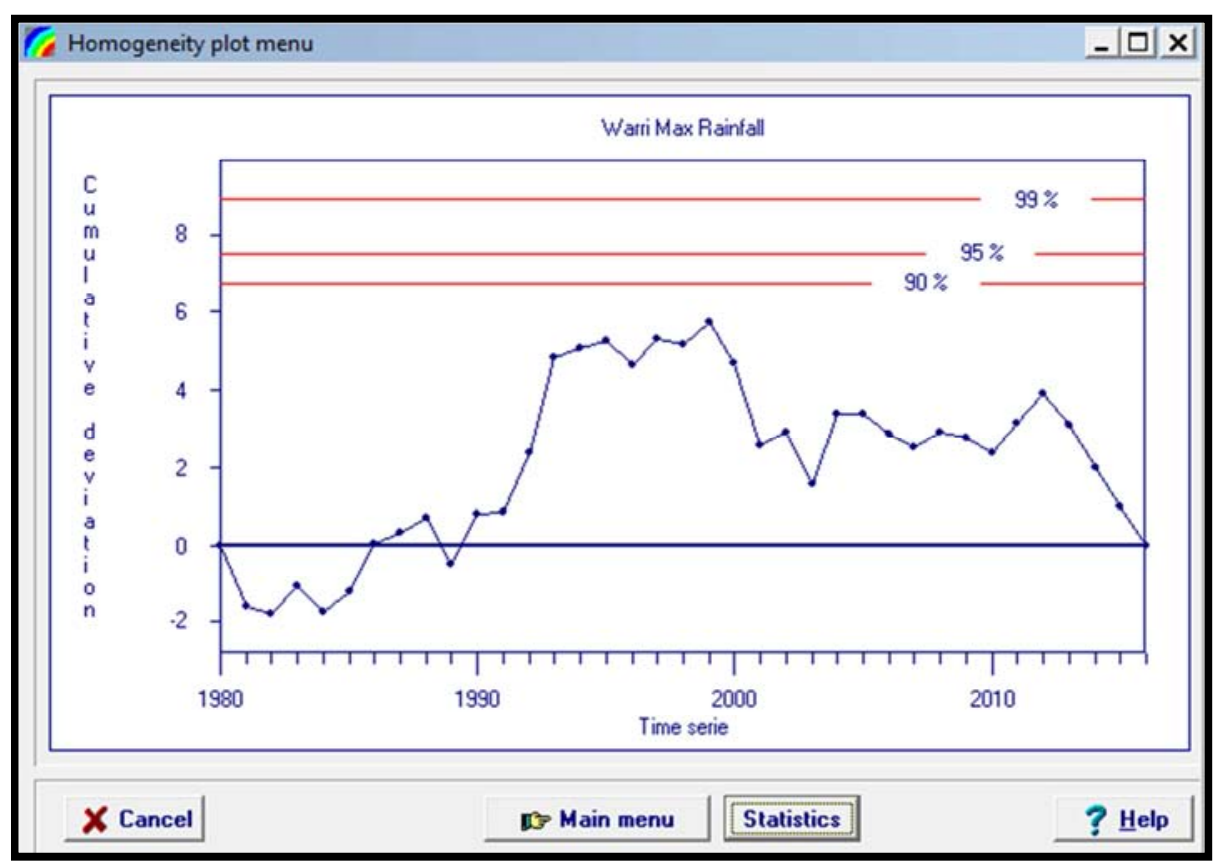

Figure 12. Homogeneity test of Warri rainfall data.

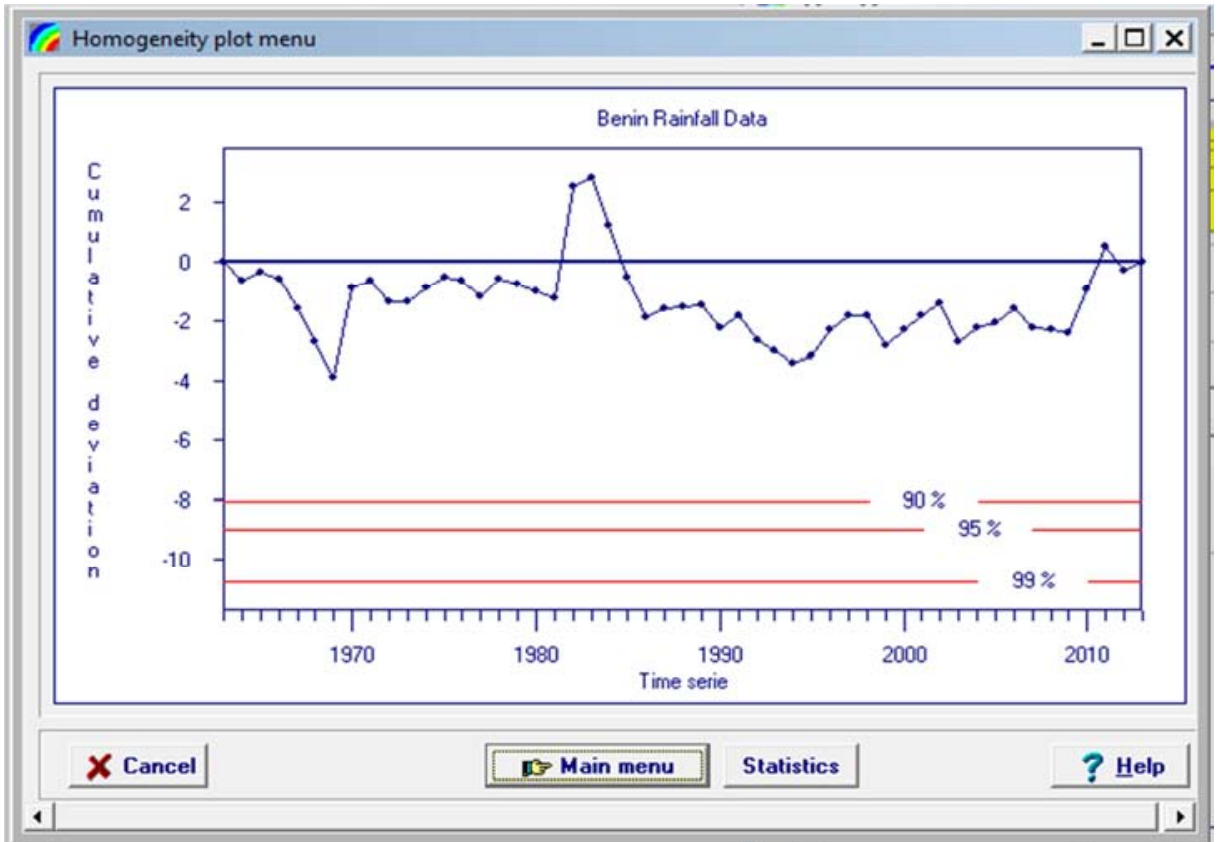

Figure 13. Homogeneity test of Benin City rainfall data.

Results of Figure 12 and Figure 13 shows that the data point fluctuate around the zero center line an indication that the rainfall data are statistically homogeneous. To further confirm that the rainfall data are statistically homogeneous, test of hypothesis was done as follows;

H0: Data are statistically homogeneous
H1: Data are not homogeneous

The null and alternate hypothesis were tested at $90 \%, 95 \%$ and $99 \%$ confidence interval that is $0.1,0.05$ and 0.01 degree of freedom and results obtained are presented in Figure 14 and Figure 15 respectively 


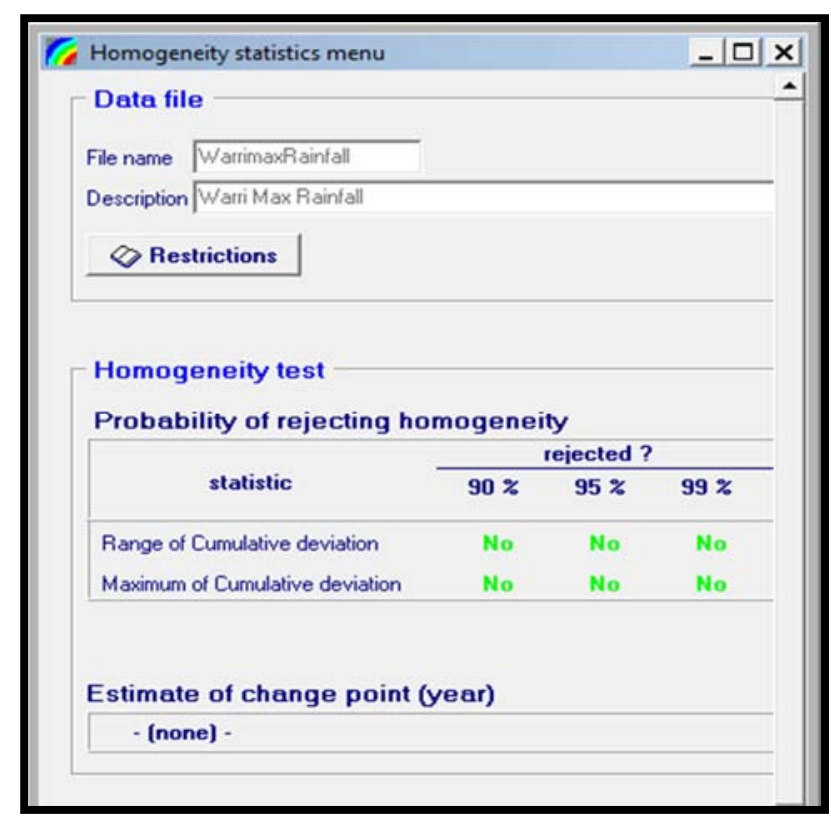

Figure 14. Homogeneity statistics of Warri rainfall data.

\begin{tabular}{|c|c|c|c|}
\hline \multicolumn{2}{|l|}{ T. Homogeneity statistics menu } & \multicolumn{2}{|c|}{ 口回 $x$} \\
\hline \multicolumn{4}{|l|}{ Data file } \\
\hline \multicolumn{4}{|l|}{ File name BeninRainfall } \\
\hline \multicolumn{4}{|l|}{ Description Benin Rainfall Data } \\
\hline \multicolumn{4}{|l|}{$\Theta$ Restrictions } \\
\hline \multicolumn{4}{|c|}{ Homogeneity test } \\
\hline \multicolumn{4}{|c|}{ Probability of rejecting homogeneity } \\
\hline \multirow[b]{2}{*}{ statistic } & \multicolumn{3}{|c|}{ rejected? } \\
\hline & $90 \%$ & $95 \%$ & $99 \%$ \\
\hline Range of Cumulative deviation & No & No & No \\
\hline Maximum of Cumulative deviation & No & No & No \\
\hline \multicolumn{4}{|c|}{ Estimate of change point (year) } \\
\hline \multicolumn{4}{|l|}{ - [none] - } \\
\hline & OK & & ? Help \\
\hline
\end{tabular}

Figure 15. Homogeneity statistics of Benin City rainfall data.

From the result of Figure 14 and Figure 15, the null hypothesis ( $\mathrm{H} 0)$ was accepted, and it was concluded that the rainfall data collected from Akure and Calabar, are statistically homogeneous at $90 \%, 95 \%$ and $99 \%$ confidence interval that is $0.10,0.05$ and $0.01 \mathrm{df}$.

On whether the rainfall data employed in this research are normally distributed, normality test was conducted. In addition to checking the distribution of the data, normality test was also aimed at selecting the most appropriate model for trend detection and estimation. If the data are normally distributed then parametric model such as least square linear regression will be most appropriate for trend detection and estimation otherwise non-parametric model such as MannKendall and Thiel Sen's slope estimation will be employed for detection and estimation of trend in the data.

For normality the histogram of the rainfall data collected from Warri and Benin City should assumed the bell shaped configuration otherwise, it is concluded that the data are not normally distributed. The histogram of the rainfall data collected from Warri and Benin City are presented in Figure 16 and Figure 17.

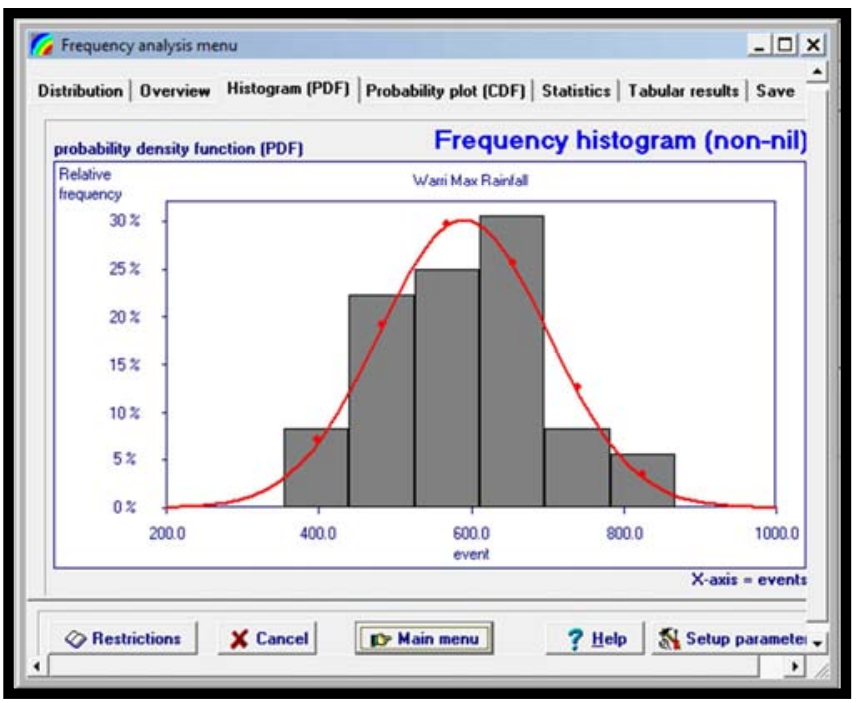

Figure 16. Histogram of Warri rainfall data.

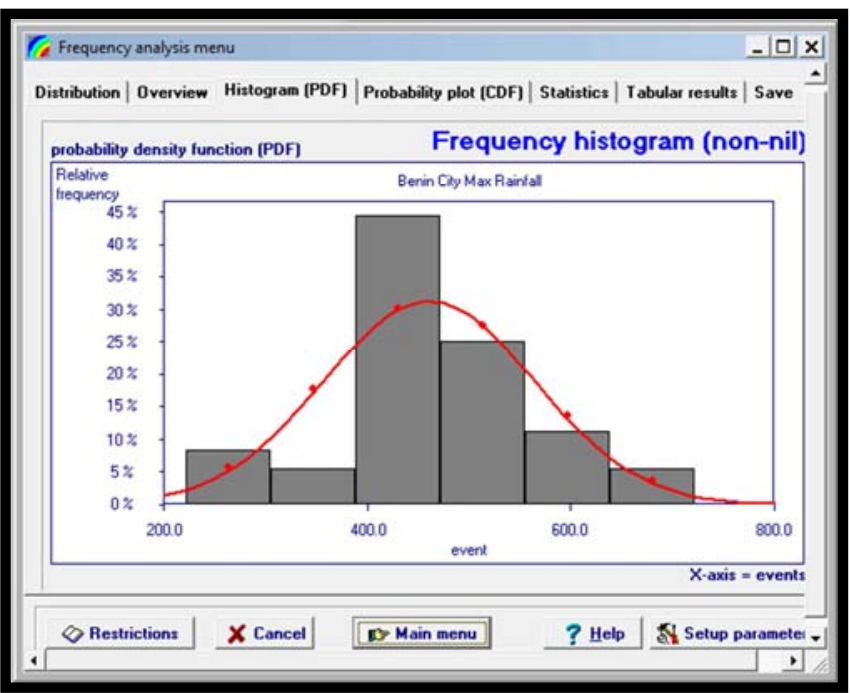

Figure 17. Histogram of Benin City rainfall data.

From the result of Figure 16 and Figure 17, it was observed that the rainfall data from Warri and Benin City are not normally distributed which is expected for most climatic variables owing to their stochastic nature. To conclude the assumption of normality, statistical hypothesis were formulated as follows

H0: The data are normally distributed

H1: The data are not normally distributed

To validate the strength of the null hypothesis, normal 
probability analysis software was employed to test the normality behaviour of the rainfall data at $99 \%$ confidence interval ( 0.01 degree of freedom). Result of the statstical analysis is presented in Figure 18 and Figure 19.

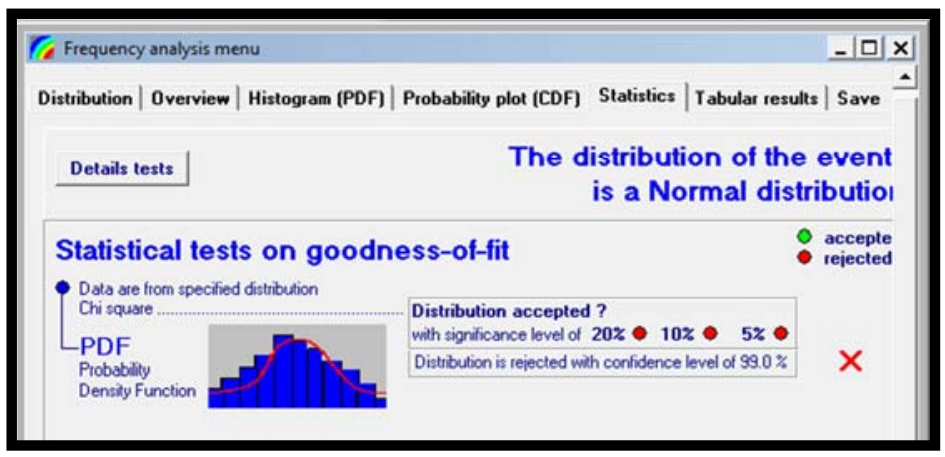

Figure 18. Normality test of Warri rainfall data.

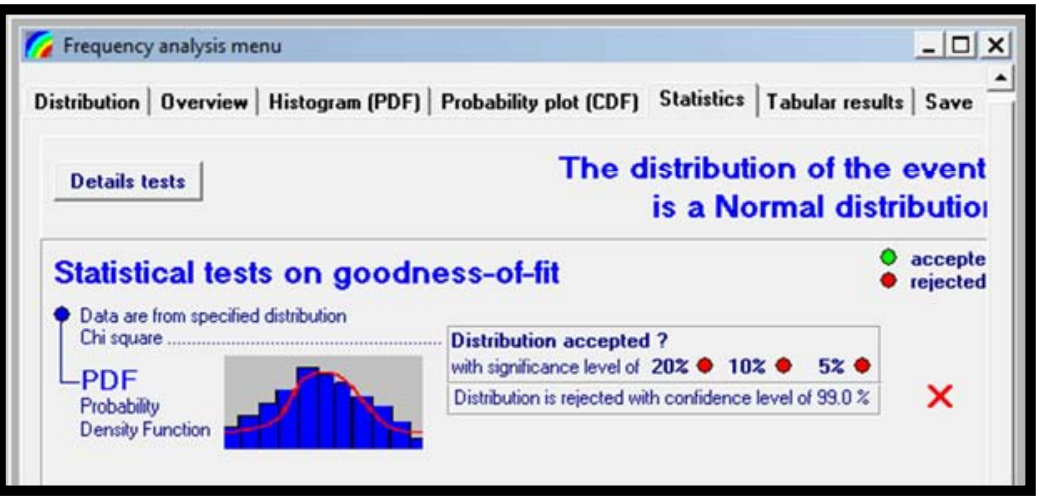

Figure 19. Normality test of Benin City rainfall data.

Result of Figures 18 and 19 revealed that rainfall data from Warri and Benin City are not normally distributed at $99 \%$ confidence interval. The implication is that non-parametric test will be most suitable in detecting and estimating the magnitude of trend associated with the data.
To perform the non-parametric analysis, the Mann-Kendall Trend test was employed. The test was conducted using Environmental Data Analysis Software (ProUCL). Results of the test are presented in Figure 20 and Figure 21

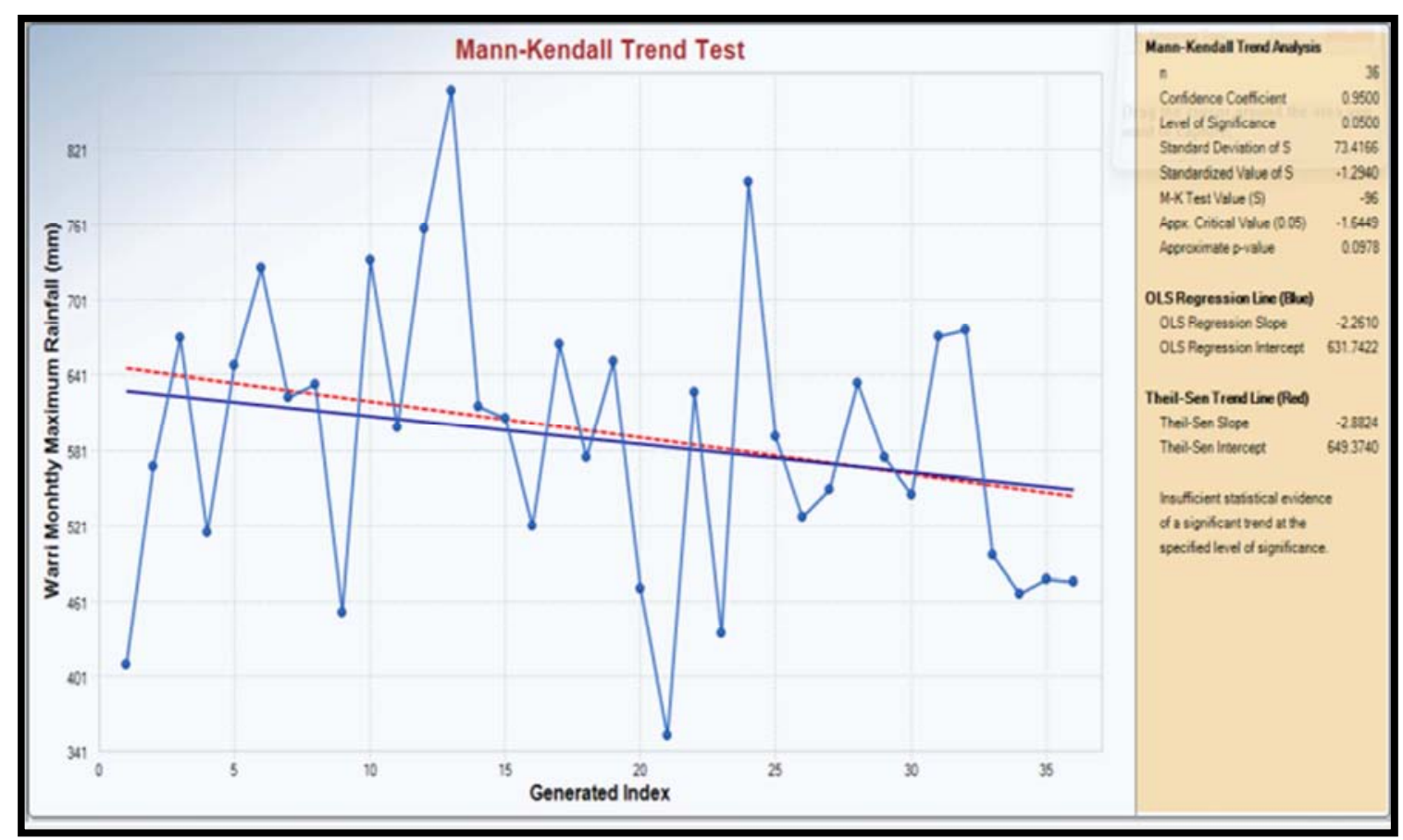

Figure 20. Mann-Kendall trend test of Warri rainfall data 


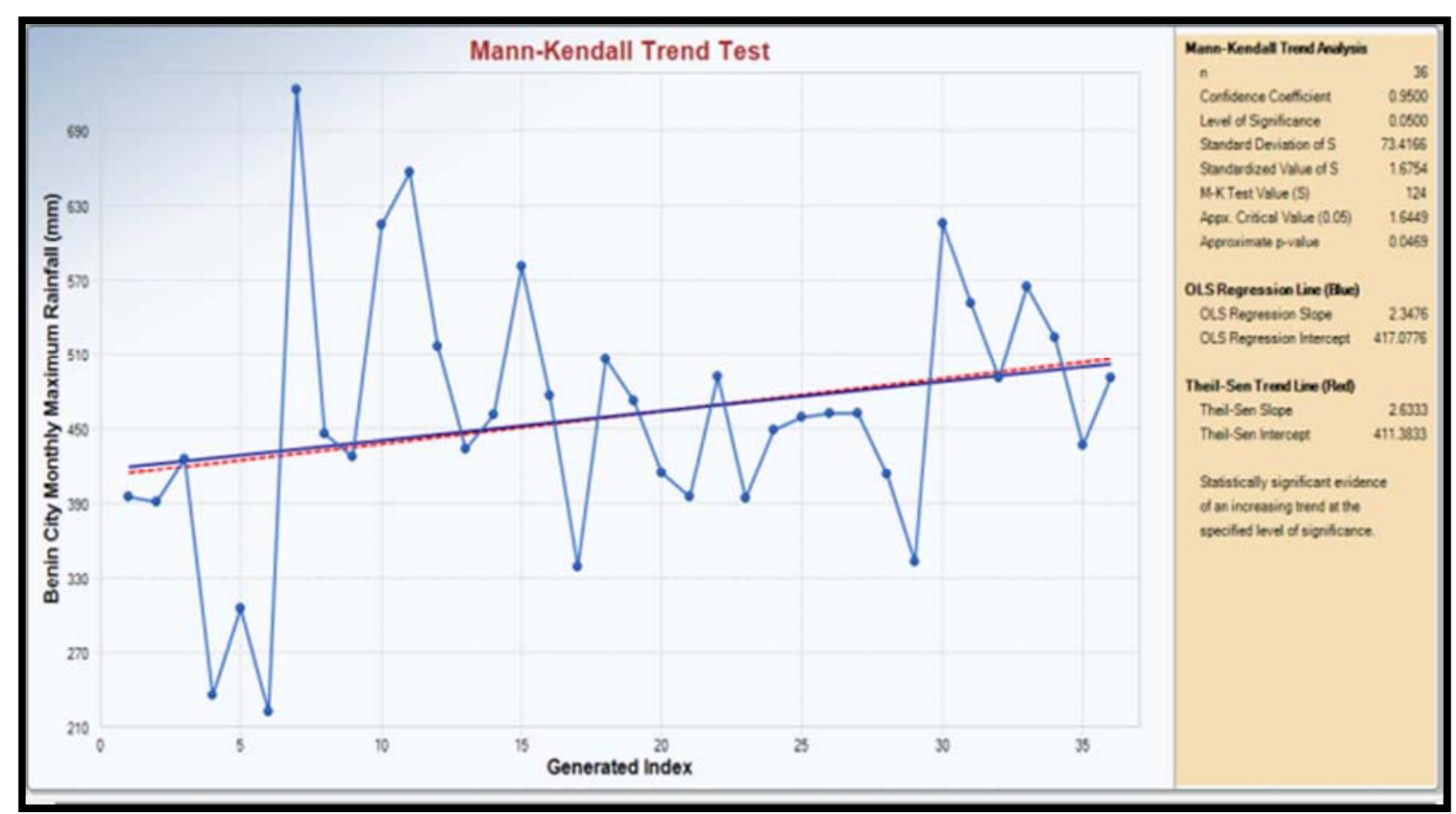

Figure 21. Mann-Kendall trend test of Benin City rainfall data

The summary result of Figure 20 and Figure 21 is presented in Table 2

Table 2. Summary of trend analysis of rainfall data from Warri and Benin City

\begin{tabular}{llll}
\hline S/No & Rainfall data location & Nature of Trend & M-K Test Value (S) \\
\hline 1 & Warri & Insufficient statistical evidence of a significant trend & -96 \\
2 & Benin City & Statistical significant evidence of an increasing trend & +124 \\
\hline
\end{tabular}

From the results of Table 2, it was observed that there was insufficient statistical evidence of a significant trend in rainfall data collected from Warri. That was not so with rainfall data from Benin City as the non-parametric test result revealed a statistical evidence of an increasing trend with $\mathrm{M}$ $\mathrm{K}$ value index of +124

\section{Conclusion}

The focus of the present study was to analyze time series data such as rainfall collected from Warri and Benin City using different time series analysis procedures. Based on the overall results, the following conclusions were made: Expectation maximization algorithm was highly effective for the infilling of missing climatic data such as rainfall. It was observed in all cases that the null hypothesis of missing completely at random was satisfied since p- value was always greater than 0.05 . Based on the outcome of the outlier detection analysis, it was concluded that climatic data used for this present analysis are devoid of possible outliers. Results of normality test further support the existing claim that climatic data are not always normally distributed owing to their stochastic nature. Finally, the non-parametric MannKendall test statistics was useful in detecting and estimating the nature of trend associated with the rainfall data from Warri and Benin City

Although, there is an insufficient statistical evidence of a significant trend in the rainfall data from Warri, the Mann-
Kendall (M-K) test value was computed as -96 which indicate a decreasing rainfall trend over time. For Benin City, statistical significant evidence of an increasing trend was observed and the calculated $\mathrm{M}-\mathrm{K}$ test value was observed to be +124 . The implication is the likelihood of flood with time. Based on the result, water resources manager should begin to plan on how to prevent such occurrence through the design and construction of water carrying structures including effective urban and regional planning

\section{References}

[1] Alli, A. A.; Oguntunde, P. G.; Olufayo, A. A., and Fasinmirin, J. T (2012), Implications of Trends and Cycles of Rainfall on Agriculture and Water Resource in the Tropical Climate of Nigeria, Special Publication of the Nigerian Association of Hydrological Sciences, pp: 188-200.

[2] Hameed, K. H. and Rao, A. R., (2008): A modified MannKendall trend test for autocorrelated data, Journal of Hydrology, vol. 204, (1-4), pp: 182-196.

[3] Ifabiyi, I. P.1 and Ojoye, S (2013), Rainfall Trends in the Sudano-Sahelian Ecological Zone of Nigeria, Earth Science Research; Vol. 2(2), pp: 194-202.

[4] Levi, D. B.; Julie, E. K.; Olsen, J. R.; Pulwarty, R. S.; Raff, D. A.; Turnipseed, D. P.; Webb, R. S and Kathleen D. W (2009); Climate Change and Water Resources Management: A Federal Perspective, circular 1331, pp: 1-72. 
[5] Raes, D; Willens, P and Gbaguidi (2006), Rainbow - A software package for analyzing data and testing the homogeneity of historical data sets, vol. 1, pp: 1-15.

[6] Shishutosh, B.; Nitin, M.; Ng, A. W. M and Perera, B. J. C (2013), Rainfall trend and its implications for water resource management within the Yarra River catchment, Australia, Hydrological Processes, Vol. 27, Issue 12, pp: 1727-1738

[7] Solomon, S., Qin, D., Manning, M., Chen, Z., Marquis, M., Averyt, K. B., Tignor, M., and Miller, H. L., (2007), Intergovernmental Panel on Climate Change, Climate change 2007: The physical science basis. Contribution of Working Group I to the Fourth Assessment Report of the Intergovernmental Panel on Climate Change, eds.: Cambridge, United Kingdom, Cambridge University Press. Pp: 1-72.
[8] Tayanç, M. And Toros, H., 1997: Urbanization effects on regional climate change in the case of four large cities of Turkey. Climatic Change, 35, 4, 501-524.

[9] Turgay, P and Ercan, K (2006), Trend analysis in Turkish precipitation data, Hydrological Processes Journal, vol. 20, pp: 2011-2026.

[10] Webb, B. W., (1996): Trends in stream and river temperature. Hydrological Processes, vol. 10 (2), pp: 205-226.

[11] Xu, Z. X.; Takeuchi, K. and Ishidaira, H., (2003): Monotonic trend and step changes in Japanese precipitation. Journal of Hydrology, vol. 279 (1-4), pp: 144-150. 